\title{
Curricular Contagion: A Case Study in Curriculum Development, Distribution, and Adoption
}

\author{
Harrigan, James ${ }^{\mathrm{a}}$; Yonk, Ryan $^{\mathrm{b}}$ and Mason, Neal ${ }^{\mathrm{c}}$ \\ ${ }^{a}$ Institute of Political Economy, Utah State University, USA, ${ }^{b}$ Department of Economics \\ and Finance, Utah State University, USA, ${ }^{\mathrm{c}}$ Strata Policy, USA.
}

\begin{abstract}
The collaborative contagion model is a byproduct of a three-year endeavor to identify and address curricular deficiencies in business ethics and entrepreneurship (BE\&E) courses. Designed to increase curriculum adoption using professional educators' established networks, the model combines a series of four-day disruptive innovation workshops with an online forum to promote collaboration in the design of $B E \& E$ materials, and to provide ongoing support for educators with unique contextual constraints. Our primary goal in developing the collaborative contagion model was to create a framework through which teachers could prototype, refine, and distribute $B E \& E$ course materials at no monetary cost. Given the variety of participants invited to the disruptive innovation workshops, we expected to produce curricular materials that incorporated a wide array of perspectives and experiences relating to BE\&E instruction. After our first year of workshops, $20 \mathrm{~K}-12$ and 20 higher education participants helped formulate 10 modules and 60 grade-specific K-12 lesson plans. Through the process, we have established pilot programs at 13 separate institutions, and built partnerships with seven organizations. In addition to providing educators with professional development opportunities and an enhanced academic network, we conclude that the collaborative contagion model promotes improved curriculum quality, and increases the likelihood of curriculum implementation.
\end{abstract}

Keywords: Collaborative curriculum design; professional development; disruptive innovation workshops; curriculum distribution; networking. 


\section{Introduction}

Since 2013, we have worked to develop a novel curriculum that will challenge the status quo in the design and delivery of business ethics and entrepreneurship (BE\&E) courses. Building from previous work by Fawson et al. (2015), which assessed innovative models for gathering input from academic networks using targeted colloquia and workshops, we have begun implementing a new model for curriculum development and distribution called a collaborative contagion model.

Adding to well-studied approaches of collaborative curriculum design (CCD), teacher design teams (TDTs), and course design intensives (CDIs), the contagion model is designed to aid in the adoption of curricula using established professional and educational networks. Our main goal has been to create a framework through which teachers can prototype, refine, and distribute quality BE\&E course materials free of charge. After hosting a series of four-day disruptive innovation workshops with participants from across the United States, we developed on an online forum for generating, hosting, revising, and rapidly distributing modules for BE\&E courses. The academic network built through this process, consisting of 13 pilot programs and seven institional partnerships, shows promise of improving both curricular quality and rates of adoption. This purpose of this paper is to summarize the literature behind the collaborative contagion model, track the model's early implementation, and explore its successes and areas for improvement.

\section{Relevant Literature}

We divide the literature behind the contagion model into four groups. The first group focuses on the benefits of CCD in teachers' professional development and in promoting a collaborative culture among faculty and staff. The second highlights potential obstacles between curriculum development and implementation, suggesting ways to increase the likelihood of implementation. The third introduces the collaborative contagion model. The final group compares CDIs with an integral part of the contagion model: the disruptive innovation workshop (DIW).

\subsection{Benefits of CCD for Teachers and Educational Faculty}

Multiple studies show how collaborative curriculum design (CCD) aids in the professional development and learning of teachers (Voogt et al., 2011; Drits-Esser \& Stark, 2015; Clark \& Hollingsworth, 2002). Teachers who assist with curriculum development show "increased self-confidence, increased pedagogical content knowledge, a deeper understanding of subject matter content, refined ideas of curriculum development in their 
personal practice, and perceptions of good teaching and being a good teacher" (Dritts-Esser $\&$ Stark, 2015, p. 2). The collaborative process also provides teachers a means for engaging peers and experts in an environment that broadens teachers' perspectives and builds leadership skills required for curriculum implementation (Voogt et al., 2011, p. 1243).

Advantages for individual teachers aside, the educational climate at an institution stands to benefit from CCD (Burrell et al., 2015). Robert Rothieaux (2015), the facilitator of a new, collaboratively-built MBA curriculum at Hamline University suggests that the "design and implementation of innovative curricula can provide a reason and means to break down barriers and create a more collaborative culture and climate" (p. 124). To this end, Rothieaux (2015) encourages bringing faculty, staff, and administrators, who generally "have little interest or reason for true collaboration," into the process (p. 124). Collaboration, in this sense, is a catalyst for greater knowledge sharing and interaction among faculty.

\subsection{Bridging the Gap between Curriculum Development and Implementation}

A primary challenge for curriculum designers is bridging the gap between curriculum development and implementation. Ideally, the professional development benefits from CCD would translate into improved classroom practice, and potentially, enhanced student outcomes. In reality, professional development often falls short of changing classroom practice. During a workshop, teachers have to navigate unfamiliarity with the design process, new pedagogical methods, and novel subject matter, thereby developing a host of new skills and knowledge (Huizinga et al., 2014). Unfortunately, teachers often return to environments that do "not always support classroom implementation of the newly learned knowledge and skills" (Voogt et al., 2011, p. 1236; Guskey, 2000). Without adequate support during and after the curriculum design process, it is unlikely teachers will experience anything more than short-term teaching changes (Clark \& Hollingsworth, 2002).

Clark and Hollingsworth's Interconnected Model of Professional Growth (IMPG) elucidates three domains in which teachers can achieve long-term change through collaborative curriculum design: (a) the personal domain (in which teachers change their knowledge, beliefs and attitude); (b) the domain of practice (in which teachers change via professional experimentation); and (c) the domain of consequence (in which the collaborative process produces salient outcomes for teachers and/or students) (2002). A fourth domain in the model, known as the external domain, provides teachers with external sources of information or stimuli. Enactment and reflection in one domain may have an impact on others (Clark \& Hollingsworth, 2002, p. 951). Change through these domains can lead to simple, short-term teaching changes, or long-term professional growth. The realization of the latter depends on, among other things, the level of ongoing support from 
colleagues and administration, resources and equipment, and the broader context in which teachers work (Clark \& Hollingsworth, 2002).

Researchers have investigated the support needs of curriculum designers required for curriculum adoption (Huizinga et al., 2013; Voogt et al., 2011). In addition to limitations on time and knowledge, teachers often lack the design expertise required for curriculum development (Huizinga et al., 2013, p. 34). Incorporating design specialists into TDTs enables teachers to apply their knowledge, skills, and contextual understanding to content and pedagogy efficiently. Another option is to provide teachers with "existing or exemplary curriculum materials...to help the team define the goals and design task" (Voogt et al., 2011, p. 1243). In any case, ongoing support and guidance by external facilitators and specialists improved teachers' overall learning during the design process (Voogt et al, 2011). Voogt et al. (2011) also discuss the importance of maintaining an explicit focus on implementation during the design process, but curriculum implementation ultimately hinges on 'teachers' ownership of and their knowledge about reform ideas" (Huizinga et al., 2013, p. 33). In other words, teachers are more likely to adopt curriculum changes in which they are involved (Dritts-Esser \& Stark, 2015, p. 3).

Realizing the support needs required for curriculum adoption, we have built two follow-up workshops into our BE\&E curriculum design process. We designed these conferences to provide ongoing support for unforeseen contextual challenges, and to build the network in a continuous fashion. Additional support is provided through a curriculum specialist at Utah State University and a website to cultivate a community of curriculum adopters.

\subsection{Description of the Contagion Model}

Sorenson et al. (2005) note that "knowledge spreads from its source not in concentric circles, but along conduits laid by social connections" (p. 4). New innovations are adopted in a manner that resembles an epidemic spreading through a population, "growing slowly at first, then accelerating rapidly, and finally slowing to reach some asymptotic saturation level" (Sorenson et al., 2005, p. 3). Social connections and proximity to the original source affect where new innovations are adopted and the rate at which they spread.

Without adopting the epidemic analogy in its entirety, our expectations for the collaborative contagion model share in many aspects of Sorenson et al.'s (2005) description of knowledge spreading. Curricular contagion begins at disruptive innovation workshops (DIWs), and relies on the efforts of workshop attendees and their own individual networks. After the workshops, which are designed in part to create a working community of conference attendees, we provide support for teachers to refine, adopt, and share developed 
materials with their colleagues. Deploying a curriculum through established networks enables us to reach a variety of new faculty, students, entrepreneurs, and policy makers.

\subsection{Comparing and Contrasting CDIs and DIWs}

Developed by Oxford Brookes University in 2003 for large-scale e-learning applications, course design intensives (CDIs) promote innovation and networking through curriculum design workshops. In a span of three to four days, CDIs yield tangible course materials as output (Dempster et al., 2012). In a program evaluation, Dempster et al. (2012) describe how CDIs utilize extended teams alongside assistance from technologists, curriculum specialists, educational developers and subject librarians (p. 137). CDI's focus explicitly on cross-disciplinary networking, using "multiple program teams working in parallel..." (Dempster et al., 2012, p. 137). Instead of leaving lecturers to their "usual subject-focused autonomy," CDIs encourage participants to work collaboratively at the program level, thereby engaging a wider array of stakeholders with various skills and experiences "to confront and to engage with alternative and better conceptions and practices" (Dempster et al., 2012, p. 136). Dempster et al. (2012) measured CDI success using the following variables: tangible deliverables, confidence and collective ownership of developed materials, networking beyond department colleagues, and conceptual and pedagogical changes for lecturers (pp. 143-144).

DIWs share many foundational ideas with CDIs. Both workshops aim to produce tangible output in the form of modules, with another expressed goal of broadening participants' networks. Like CDIs, DIWs use parallel sets of extended teams, equipped with experts to analyze theory, discuss technical obstacles and solutions, and draft modules. Dempster et al.'s (2012) measures for successful CDIs apply equally to our internal measures for gauging curricular contagion.

Unlike DWIs, CDIs "are not a tactic to initiate change or raise awareness" (Dempster et al., 2012, p. 137). We intend our DIWs to change the delivery and design of BE\&E courses through heightened awareness and outreach. The contagion effect depends on participants' willingness and ability to share resources and improve BE\&E course quality.

The composition of teams also differs between CDIs and DIWs. The CDIs reviewed by Dempster et al. (2012) assembled teams from faculty and staff at a single university. Our DIWs, on the other hand, hosted educators from multiple institutions ranging in size, approach, scope, and location. It was our goal to create an environment that would address a broad range of programmatic needs heretofore inhibited by geographic and institutional siloing, thereby encouraging nationwide curriculum adoption. 


\section{The Process Behind the Contagion Model}

We began the process of developing the contagion model after researching potential gaps in existing BE\&E curricula. After surveying 170 BE\&E course syllabi throughout the United States, we found that new offerings in these subjects had taken on a variety of forms, transitioning from appendages of more established disciplines into discrete, stand-alone courses. Entrepreneurship courses often considered only new venture startup, without broader discussion of what it meant to be entrepreneurial, or the benefits of entrepreneurship for society. Different AACSB-accredited schools offered courses under the business ethics banner that contained completely disparate content (Fawson et al., 2015). Some business ethics courses emphasized a foundation in classical philosophy, whereas others focused exclusively on a legal-positivistic approach, stressing adherence to established codes and policies. While we have always viewed educational flexibility and license in a positive light, our concern was that such disparity might leave business students ill-equipped to navigate an array of moral dilemmas faced in the workplace.

We designed the innovation workshops to utilize the dispersed knowledge of participants, and to draft modules that would address these and other shortcomings in existing courses, consolidating, to a degree, BE\&E curricula being offered throughout the country. Our goal was to initiate a process of collaboration and refinement that would culminate in usable, standards-ready materials that could be shared and adopted at no monetary cost to teachers.

While recruiting attendees for the innovation workshops, we sought a balance of individuals within our network and others with little or no connection at all. Our academic network and social media presence helped us identify individuals within business schools, philosophy departments, K-12 teaching positions, and administration, all of whom would, we hoped, make significant contributions in developing new course materials. Although participants' notoriety and roles varied, each demonstrated a shared desire to effect a positive change in the current orientation of ethics and entrepreneurship courses.

Prior to the innovation workshops, we asked participants to submit any readings that could provide a baseline for subject matter competence, and would facilitate conversation among participants on common difficulties in teaching BE\&E. After compiling and distributing the readings, we asked that participants read all materials before coming to the workshops.

At the beginning of each day during the workshops, we used design-thinking activities to encourage new ways of thinking about BB\&E, and to overcome barriers to participation. Round-table discussions at the conference helped teachers and administrators establish the current state of the courses, and navigate pedagogical and institutional obstacles they face when trying to innovate in their classrooms or utilize a new curriculum. 
We encouraged ownership of developed materials by asking workshop participants to contribute activities and lesson plans from their experience. As they did, we constructed prototypes. These prototyped lesson plans were then posted online for educators to use freely, revise, and distribute to their colleagues. The website continues to provide a virtual medium for ongoing collaboration, keeps a log of new participants in the growing network, and tracks where curricular adoption takes place.

At the end of the workshops, participants were surveyed about the knowledge they gained from the experience and their ongoing commitment to implement modules and lesson plans in their various faculty positions across the United States. We provided post-workshop support for teachers in the form of pre-prepared course evaluation tools and surveys for future BE\&E courses.

\section{Results \& Early Indicators of Success}

We worked with a total of $20 \mathrm{~K}-12$ and 20 higher education participants at our first two disruptive innovation workshops. Through these events, we established pilot programs at 13 separate institutions, and built partnerships with seven organizations. Participants produced five general lesson ideas/activities, from which we have built 10 modules and 60 gradespecific K-12 lesson plans.

Many of the participants attending the conference had little experience with design activities and lacked the requisite vocabulary for understanding and producing novel curricular components. The discomfort was especially pronounced for K-12 educators. One K-12 teacher said, "There was a bit of disconnect between the university professors and the 'ground truth' of K-12 educators." Another K-12 teacher expressed concern that "the majority of the conversations seemed about philosophy rather than pedagogy," making it difficult to participate. While professors thrived in the open-ended, early curricular discussions, several K-12 educators wanted more definitive pedagogical items to discuss.

Very few of our participants came from outside the United States. In the future, we hope to field test our collaborative contagion model in foreign networks. We are presently providing support for educators who attended the workshops and others who have been introduced to the curriculum via social networks.

\section{Conclusions}

Citing ideas from economist Kenneth Arrow, Sorenson et al. (2005) describe how "the generation of new knowledge often requires substantial investment in research and development, but the repeated application of this knowledge, once produced, entails little if 
any incremental cost" (p. 1). We designed our collaborative model to help educators develop and share quality BE\&E materials. Ongoing refinement of modules among participants in different professional networks has produced multiple prototypes of lesson plans from which teachers can choose and adapt to various situational demands. In our model, teachers also have incentives to participate: better lesson plans, professional development hours, network building, and program development ideas. Utilizing the dispersed knowledge of participants, the contagion model has helped us avoid knowledge sharing limitations and the siloing of content along geographic boundaries, ensured essential coverage of foundational principles, and encouraged wider curricular adoption.

\section{References}

Burrell, A.R., Cavanagh, M, Young, S., \& Carter, H. (2015). Team-based curriculum design as an agent of change. Teaching in Higher Education, 20(8), 753-766.

Clarke, D., \& Hollingsworth, H. (2002). Elaborating a model of professional growth: Teaching and Teacher Education, 18, 947-967.

Dempster, J.A. (2012). An academic development model for fostering innovation and sharing in curriculum design. Innovations in Eucation and Teaching International, 49(2), 135-147.

Drits-Esser, D., \& Stark, L.A. (2015). The Impact of Collaborative Curriculum Design on Teacher Professional Learning. Electronic Journal of Science Education, 19(8), 1-27.

Fawson, C., Simmons, R., \& Yonk, R. (2015). Curricular and Programmatic Innovation at the Intersection of Business Ethics and Entrepreneurship. In S. Hoskinson, \& D. Kuratko (Eds.), Advances in the Study of Entrepreneurship, Innovation and Economic Growth, Volume 25: The Challenges of Ethics and Entrepreneurship in the Global Environment (pp. 109-130). Bingley, UK: Emerald Group Publishing Limited.

Guskey, T.R. (2000). Evaluating professional development. Thousand Oaks, CA: Corwin Press.

Huizinga, T., Handelzalts, A., Nieveen, N., \& Voogt, J.M. (2014). Teacher involvement in curriculum design: need for support to enhance teachers' design expertise. Journal of Curriculum Studies, 46(1), 33-57.

Routhieaux, R.L. (2015). Fostering Integrated Learning and Faculty Collaboration through Curriculum Design: A Case Study. Journal of Curriculum and Teaching, 4(1), 122-132.

Sorenson, O., Rivkin, J., \& Fleming, L. (2005). Complexity, Networks and Knowledge Flow. Paper presented at the DRUID Tenth Anniversary Summer Conference of 2005 on the Dynamics of Industry and Innovation: Organizations, Networks and Systems, Copenhagen, Denmark. Retrieved from http://www.druid.dk/uploads/tx_picturedb/ds2005-1599.pdf.

Voogt, J., Westbroek, H., Handelzalts, A., Walraven, A., McKenney, S., Pieters, J., \& de Vries, B. (2011). Teacher learning in collaborative curriculum design. Teaching and Teacher Education, 27(8), 1235-1244. 